\title{
REGULATIONS GOVERNING CLINICAL TRIALS IN INDIA, EUROPE AND USA- A COMPARATIVE STUDY
}

Available online at www.ijdra.com

REVIEW ARTICLE

Agarwal Pooja*, Priyanka B

Faculty in Dept. of Pharmaceutical Management and Regulatory Affairs, Geethanjali College of Pharmacy, Cheeryal(V), Keesara (M), Medchal Dist., Hyderabad, Telangana, India 501301

*Corresponding Author's E-mail: p_agarwal89@yahoo.co.in

DOI:https://doi.org/10.22270/ijdra.v5i1.195

\begin{abstract}
Clinical trials are research studies that explore whether a medical strategy, treatment, or device is safe and effective for humans. These studies also may show which medical approaches work best for certain illnesses or groups of people. The purpose of clinical trials is research, so the studies follow strict scientific standards. These standards protect patients and help produce reliable study results. Clinical trials are one of the final stages of a long and careful research process. The process often begins in a laboratory (lab), where scientists first develop and test new ideas. If an approach seems promising, the next step may involve animal testing. This shows how the approach affects a living body and whether it is harmful. However, an approach that works well in the lab or animals does not always work well in people. Thus, research in humans is needed.
\end{abstract}

Keywords: Clinical trials, humans, Phase 1, research, standards.

\section{INTRODUCTION}

Clinical trials explore how a treatment reacts in the human body and are designed to ensure a drug is tolerated and effective before it is licensed by regulatory authorities and made available for use by doctors. Studies vary in their primary goal or endpoint (i.e. the most important outcome of the trial), the number of patients involved, and the specifics of the study design. However, all clinical studies conform to a strict set of criteria to protect the patients involved and to ensure rigorous evaluation of the drug. (1)

For safety purposes, clinical trials start with small groups of patients to find out whether a new approach causes any harm. In later phases of clinical trials, researchers learn more about the new approach's risks and benefits.

A clinical trial may find that a new strategy, treatment, or device

- improves patient outcomes;

- offers no benefit; or
- Causes unexpected harm. (2)

The goal of clinical trials is to create an environment that is favourable to conducting clinical trials, with the highest standards of safety for participants and increased transparency of trial information.

The regulation will require:

- consistent rules for conducting clinical trials

- information on the authorization, conduct and results of each clinical trial carried out to be publicly available.

This will increase the efficiency of all trials with the greatest benefit for those conducted in multiple Member States. It aims to foster innovation and research, while helping avoid unnecessary duplication of clinical trials or repetition of unsuccessful trials.

\section{Drug Development Process}

- New medicines originate in the laboratory where researchers identify, 
isolate and study thousands of molecules for their potential as future therapies.

- Once a candidate molecule (compound) has been identified in the laboratory, it is subjected to rigorous pre-clinical testing (in the laboratory and/or in animals) to assess its chemical, biological and toxicological properties (how harmful it is).

- These pre-clinical tests allow researchers a snapshot of whether a compound may have pharmacological activity.

- If results of pre-clinical studies are positive, the compound may be entered into a clinical trial program: this involves several 'phases' of study, starting with small studies usually in healthy volunteers and progressing in steps through to evaluation of the drug in people with the disease. At each phase, only those compounds that meet strict criteria for safety and effectiveness (efficacy) advance to the next phase. effective the company applies to regulatory authorities for marketing authorization (permission to sell. (3)

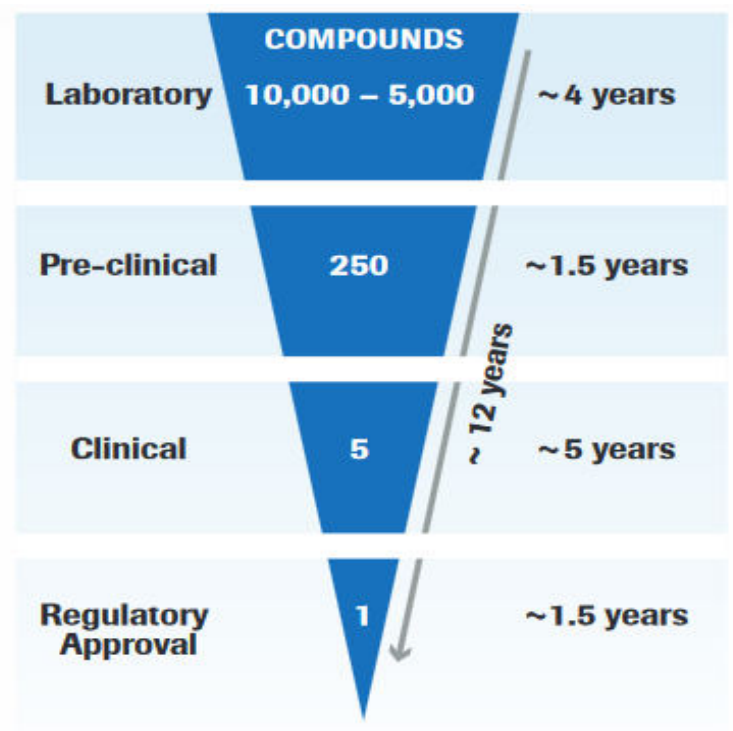

Figure 1: Drug Development Process

CLINICAL TRIAL PHASES IN INDIA, EU AND USA: (1)

- When results of clinical trials indicate the compound being studied is safe and The clinical trial phases are same in all the three countries. They are:

\section{New Drug Clinical Trials}

Downward Trend: Only 16 out of every 100 drugs that enter Phase 1 will make it to FDA approval.

\begin{tabular}{|c|c|c|c|}
\hline $\begin{array}{l}\text { 20-100 volunteers } \\
1^{\text {st }} \text { state of testing } \\
\text { in humans }\end{array}$ & $\begin{array}{l}\mathbf{1 0 0} \text { - } \mathbf{5 0 0} \text { patients } \\
\text { How well does } \\
\text { the drug work? }\end{array}$ & $\begin{array}{l}\mathbf{1 , 0 0 0}-\mathbf{5 , 0 0 0} \text { patients } \\
\text { - Drug MUST be safe } \\
\text { - Comparison with current } \\
\text { 'gold standard' treatment }\end{array}$ & $\begin{array}{l}\text { Safety surveillance in } \\
\text { 'Real-life'patients }\end{array}$ \\
\hline
\end{tabular}

Figure 2: Clinical trial phases

\section{Phase 0 clinical trial (or) pre-clinical trial}

Even though phase 0 studies are done in humans, this type of study is not like the other phases of clinical trials. The purpose of this phase is to help speed up and streamline the drug approval process.

Phase 0 studies are exploratory studies that often use only a few small doses of a new drug in a few patients. They test to find out whether the drug reaches the tumor, how the drug acts in the human body, and how cancer cells in the human body respond to the drug. The patients in these studies might need extra biopsies, scans, and blood samples as part of the study process. 
The biggest difference between phase 0 and the later phases of clinical trials is that there is no chance the volunteer will benefit by taking part in a phase 0 trial - the benefit will be for other people in the future. Because drug doses are low, there is also less risk to the patient in phase 0 studies compared to Phase I studies.

Phase 0 studies help researchers find out whether the drugs do what they're expected to do. If there are problems with the way the drug is absorbed or acts in the body, this should become clear very quickly in a phase 0 clinical trial. This process may help avoid the delay and expense of finding out years later in phase II or even phase III clinical trials that the drug doesn't act as expected to based on lab studies.

Phase 0 studies aren't used widely, and there are some drugs for which they wouldn't be helpful. Phase 0 studies are very small, often with fewer than 15 people, and the drug is given only for a short time. They are not a required part of testing a new drug.

\section{Phase I clinical trials: Is the treatment safe?}

Phase I studies of a new drug are usually the first that involve people. The main reason for doing phase I studies is to find the highest dose of the new treatment that can be given safely without serious side effects. Although the treatment has been tested in lab and animal studies, the side effects in people cannot always be predicted. These studies also help to decide on the best way to give the new treatment.

\section{Key points of phase I clinical trials}

- The first few people in the study often get a low dose of the treatment and are watched very closely. If there are only minor side effects, the next few participants may get a higher dose. This process continues until doctors find the dose that is most likely to work while having an acceptable level of side effects.

- The focus in phase I is looking at what the drug does to the body and what the body does with the drug.

- Safety is the main concern at this point. Doctors keep a close eye on the people and watch for any serious side effects. Because of the small numbers of people in phase I studies, rare side effects may not be seen until later.

- Placebos are not part of phase I trials.

- These studies usually include a small number of people (20 to 80).

- Often, people with different types of cancer can take part in the same Phase I study.

- These studies are usually done in major cancer centers.

- These studies are not designed to find out if the new treatment works against cancer.

Overall, phase I trials are the ones with the most potential risk. But phase I studies do help some patients. For those with life-threatening illnesses, weighing the potential risks and benefits carefully is key.

\section{Phase II clinical trials: Does the treatment work?}

If a new treatment is found to be reasonably safe in phase I clinical trials, it can then be tested in a phase II clinical trial to find out if it works. The type of benefit or response the doctors look for depends on the goal of the treatment. It may mean the cancer shrinks or disappears. Or it might mean there's an extended period of time where the cancer doesn't get any bigger, or there's a longer time before the cancer comes back. In some studies, the benefit may be an improved quality of life. Many studies look to see if people getting the new treatment live longer than they would have been expected to without the treatment.

\section{Key points of phase II clinical trials}

- Usually, a group of 25 to 100 patients with the same type of cancer gets the new treatment in a phase II study. They are treated using the dose and method found to be the safest and most effective in phase I studies.

- In a phase II clinical trial, all the volunteers usually get the same dose. But some phase II studies do randomly assign participants to different treatment groups (much like what's done in phase III trials). These groups may get different doses or get the treatment in different ways to see which provides the best balance of safety and effectiveness. 
- No placebo is used.

- Phase II studies are often done at major cancer centers, but may also be done in community hospitals or even doctors' offices.

Larger numbers of patients get the treatment in phase II studies, so there's a better chance that less common side effects may be seen. If enough patients benefit from the treatment, and the side effects are not too bad, the treatment is allowed to go on to a phase III clinical trial. Along with watching for responses, the research team keeps looking for any side effects.

\section{Phase III clinical trials: Is it better than what's already available?}

Treatments that have been shown to work in phase II studies usually must succeed in one more phase of testing before they are approved for general use. Phase III clinical trials compare the safety and effectiveness of the new treatment against the current standard treatment.

Because doctors do not yet know which treatment is better, study participants are often picked at random (called randomized) to get either the standard treatment or the new treatment. When possible, neither the doctor nor the patient knows which of the treatments the patient is getting. This type of study is called a double-blind study.

\section{Key points of phase III clinical trials}

- Most phase III clinical trials have a large number of patients, at least several hundred.

- These studies are often done in many places across the country (or even around the world) at the same time.

- Phase III clinical trials are more likely to be offered by community-based oncologists.

- These studies tend to last longer than phases I and II.

- Placebos may be used in some phase III studies, but they are never used alone if there is a treatment available that works.

As with other studies, patients in phase III clinical trials are watched closely for side effects, and treatment is stopped if they're too bad.

\section{Submission for FDA approval: New drug application (NDA)}

In the United States, when phase III clinical trials show a new drug is more effective and/or safer than the current standard treatment, a new drug application (NDA) is submitted to the Food and Drug Administration (FDA) for approval. The FDA then reviews the results from the clinical trials and other relevant information.

After review, the FDA may ask for more information or even require that more studies be done. This can extend the approval process to more than 5 years.

On the other hand, based on its review, the FDA can decide that the treatment is OK to be used in patients with the type of illness the drug was tested on. In this case, the new treatment often becomes the standard of care, and newer drugs must then be tested against it before being approved. However, in some cases, the clinical trials are still not over and phase IV trials continue.

\section{Phase IV clinical trials: What else do we need to know?}

Drugs approved after phase III trials are often watched over a long period of time in phase IV studies. Even after testing a new medicine on thousands of people, the full effects of the treatment may not be known. Some questions may still need to be answered. For example, a drug may get FDA approval because it was shown to reduce the risk of cancer coming back after treatment. But does this mean that those who get it are more likely to live longer? Are there rare side effects that haven't been seen yet, or side effects that only show up after a person has taken the drug for a long time? These types of questions may take many more years to answer, and are often addressed in phase IV clinical trials.

\section{Key points of phase IV clinical trials}

- Phase IV studies look at drugs that have already been approved by the FDA. The drugs are available for doctors to prescribe 
for patients, but phase IV studies are needed to answer important questions.

- May involve at most, a few tens of thousands of people.

- This is the safest type of clinical trial because the treatment has already been studied a lot and used in possibly millions of people. Phase IV is looking at safety over time.

- These studies may also look at other aspects of the treatment, such as quality of life or cost effectiveness.

\section{Phase V clinical trials}

- This translational research is designed to "move from bench to bedside". Phase V clinical trials refer to comparative effectiveness research and community-based research. Research is done on data collected. All reported uses are evaluated. Patients are not monitored. Its main focus is to determine integration of a new therapy into wide spread clinical practice.

\begin{tabular}{cccc}
\hline Phases & Dosing & Number of subjects & Main goal of clinical phase \\
\hline Preclinical & Unrestricted & Not applicable & Testing in non-humans (efficacy, toxicities, pharmacokinetics) \\
0 & Subtherapeutic & About 10 & Pharmacokinetics and pharmacodynamics \\
IA/IB & Ascending doses & $20-100$ & Dose-ranging \\
IIA/IIB & Therapeutic dose & $100-300$ & Drug efficacy \\
IIIA/IIIB & Therapeutic dose & $1000-2000$ & Therapeutic effect \\
IV & Therapeutic dose & Anyone seeking treatment & Long-term effects \\
V & No dosing & All reported use & Research on data collected \\
\hline
\end{tabular}

Figure 3: Clinical trial phases, Subjects and Goal

\section{CLINICAL REGULATORY AGENCIES - INDIA (4)}

The main regulatory agencies for clinical trials in India:

1. Ministry of Health and Family Welfare

2. Central Drug Standards Control Organization

3. Indian Council of Medical Research

4. Ministry of Chemicals and Fertilizers

\section{Ministry of Health and Family Welfare}

This is the regulatory agency, which is primarily dealing with healthcare. This government body has several bodies under its administrative control. Some of them are:

- Medical Council of India

- Dental Council of India

- Pharmacy Council of India

- Central Drug Standards Control Organization
- Hospital Services Consultancy Corporation Limited

This regulatory agency acts by prescribing the standards in order to ensure the safety, efficacy as well as the quality of the following products:

- Drugs

- Cosmetics

- Diagnostics

- Devices

In addition, this regulatory agency is also regulating the following aspects of clinical trial

- Market authorization of new drugs

- Clinical trials standards

The role of Ministry of Health and Family Welfare in a clinical trial does not stop with this alone. This body will also supervise the drug imports. Furthermore, it is this regulatory agency, which will approve the license to drug manufacture. 


\section{Central Drug Standards Control Organization}

This national regulatory authority is being operated under the 'Ministry of Health and Family Welfare'. This is the primary regulatory authority for the pharmaceuticals as well as medical devices in the nation. The Central Drug Standards Control Organization is serving the parallel function to the U.S. FDA, Japan's PMDA and European Union's EMA.

With this regulatory agency, it is the Drug Controller General of India who is regulating all the pharmaceuticals and medical devices. As such, the DCGI is being advised by two other bodies, which are:

- Drug Technical Advisory Board

- Drug Consultative Committee

As such, the whole regulatory agency has been divided into zonal offices to perform the following functions:

- Pre-licensing inspections

- Post-licensing inspections

- Post-market surveillance

- Recalls (if required)

The Central Drug Standards Control Organization is maintaining a good track record with 'World Health Organization'. At present, this agency is planning to open its international offices in China.

\section{Indian Council of Medical Research}

This is one among the oldest research bodies of the country. The Indian Government is funding this agency. The governing body of this organization is presided by the Union Health Minister. In addition, the scientific advisory board is assisting this regulatory agency. Various eminent experts in biomedical disciplines will assist ICMR in both scientific and technical matters. This regulatory agency is acting to promote biomedical research in the country and is considered as the apex body for the following activities:

- Formulation of biomedical research

- Coordination of biomedical research

- Promotion of biomedical research
ICMR is the regulatory body, which has formulated guidelines for several aspects that are relating to national health. Treatments for conditions like malaria, cancer, type 2 - diabetes and retinoblastoma have been covered by various guidelines by Indian Council of Medical Research.

The ICMR guidelines that are pertaining to the clinical trial research in the country are:

- Ethics guidelines for biomedical research: This includes the following information:

- General research principles for the research works that involve human subjects.

- Specific research principles for the research works that involve human subjects in specific areas.

- Guidelines for Good Clinical Laboratory Practices: These guidelines are aiming to elucidate the step-wise procedures that are to be followed by the laboratories for strengthening the quality of all the test results. In India, these guidelines must be taken up by all the ICMR labs, which are engaging in clinical trial research. A checklist will be prepared in order to monitor all these ICMR laboratories for compliance with ICMR guidelines.

\section{Ministry of Chemicals and Fertilizers}

This ministry in India is the primary administrative unit of the following departments:

- Department of Chemical and Petrochemicals

- Department of Fertilizers

- Department of Pharmaceuticals

The ministry of chemicals and fertilizers is involved in the following activities:

- Setting and revising the drug prices

- Maintaining the data on production/ export/import

- Enforcing and monitoring the availability of medicines

- Giving options to the parliament

- This government body is regulating the industrial policy on drugs in the country. 


\section{CLINICAL REGULATIONS IN EUROPE} (5)

The European Medicines Agency (EMA) is a European Union agency for the evaluation of medicinal products. Prior to 2004, it was known as the European Agency for the Evaluation of Medicinal Products (EMEA). The EU is currently the source of about one-third of the new drugs brought onto the world market each year.

The main regulatory agencies for clinical trials in Europe are:

\section{Committee for Medicinal Products for Human Use}

A single evaluation is carried out through the Committee for Medicinal Products for Human Use (CHMP). If the Committee concludes that the quality, safety and efficacy of the medicinal products are sufficiently proven, it adopts a positive opinion. This is sent to the European Commission to be transformed into a marketing authorization valid for the whole of the EU. A special type of approval is the Paediatric-Use Marketing Authorisation (PUMA), which can be granted for medical products intended exclusively for paediatric use.

The CHMP is obliged by the regulation to reach decisions within 210 days, though the clock is stopped if it is necessary to ask the applicant for clarification or further supporting data. This compares well with the average of 500 days taken by the U.S. Food and Drug Administration.

\section{Committee for Medicinal Products for Veterinary Use}

The Committee for Medicinal Products for Veterinary Use (CVMP) operates in analogy to the CHMP as described above.

\section{Committee on Orphan Medicinal Products}

The Committee on Orphan Medicinal Products (COMP) administers the granting of orphan drug status since 2000. Companies intending to develop medicinal products for the diagnosis, prevention or treatment of life-threatening or very serious conditions that affect not more than five in 10,000 persons in the European Union can apply for 'orphan medicinal product designation'. The COMP evaluates the application and makes a recommendation for the designation, which is then granted by the European Commission.

\section{Committee on Herbal Medicinal Products}

The Committee on Herbal Medicinal Products (HMPC) assists the harmonization of procedures and provisions concerning herbal medicinal products laid down in EU Member States, and further integrating herbal medicinal products in the European regulatory framework since 2004.

\section{Paediatric Committee}

The Paediatric Committee (PDCO) deals with the implementation of the paediatric legislation in Europe Regulation (EC) No 1901/2006 since 2007. Under this legislation, all applications for marketing authorization of new medicinal products, or variations to existing authorizations, have to either include data from paediatric studies previously agreed with the PDCO, or obtain a PDCO waiver or a deferral of these studies.

\section{Committee for Advanced Therapies}

The Committee for Advanced Therapies (CAT) was established in accordance with Regulation (EC) No 1394/2007 on advanced-therapy medicinal products (ATMPs) such as gene therapy, somatic cell therapy and tissue engineered products. It assesses the quality, safety and efficacy of ATMPs, and follows scientific developments in the field.

\section{Pharmacovigilance Risk Assessment Committee}

A seventh committee, the Pharmacovigilance Risk Assessment Committee (PRAC) has come into function in 2012 with the implementation of the new EU pharmacovigilance legislation (Directive 2010/84/EU). The Agency constantly monitors the safety of medicines through a pharmacovigilance network and EudraVigilance, so that it can take appropriate actions if adverse drug reaction reports suggest that the benefit-risk balance of a medicine has changed since it was authorized. (5) 
When the Regulation becomes applicable, it will replace the existing EU Clinical Trial Directive (EC) No. 2001/20/EC and national legislation that was put in place to implement the Directive.

The authorization and oversight of clinical trials remains the responsibility of Member States, with EMA managing the database and supervising content publication on the public website.

Key benefits of the Regulation

- Harmonized electronic submission and assessment process for clinical trials conducted in multiple Member States.

- Improved collaboration, information sharing and decision-making between and within Member States.

- Increased transparency of information on clinical trials.

- Highest standards of safety for all participants in EU clinical trials.

EU clinical trial portal and database

The portal will be the single entry point for submitting clinical trial information in the EU, which will be stored in the database. EMA will make information stored in the database publicly available subject to transparency rules.

The clinical trial application form and supporting dossier will cover all regulatory and ethics assessments from the Member States concerned. It will also include the public registration of the clinical trial and any subsequent updates.

EMA published the functional specifications for the EU portal and database to be audited in December 2014, following a public consultation:

- Functional specifications for the EU portal and EU database to be audited

The system will contain collaboration tools, workflow and document management capabilities, accessible via individual workspaces.

Sponsor workspace
A secure workspace will assist clinical trial sponsors in preparing and compiling data to submit to the database for assessment by Member States. It will allow sponsors to:

- $\quad$ search and access clinical trials;

- compile clinical trial application dossiers for new and updated trials;

- cross-reference to product documents in other clinical trials;

- supervise their own clinical trials and check progress;

- receive alerts and notifications for ongoing trials;

- record clinical trial results;

- upload documents for clinical trial application form submission;

- respond to requests for information and view deadlines;

- manage users and user roles.

Authority workspace:

A secure workspace will support the activities of Member States and the European Commission in overseeing clinical trials. It will allow Member States to:

- view application dossiers;

- manage tasks related to the assessment of clinical trials;

- collaborate within and between Member States;

- receive alerts and notifications for ongoing trials;

- download documents submitted by clinical trial sponsors;

- record inspections of sites and clinical trials.

Public website

Through the website, members of the public can access detailed information on all clinical trials conducted in the EU, in all official EU languages. The website will provide the following features:

- overview of clinical trial statistics;

- advanced search;

- download data and reports;

- site updates and announcements.

Implementation

Although the regulation was adopted and entered into force in 2014, the timing of its 
application depends on confirmation of full functionality of the EU portal and database through an independent audit. The Regulation becomes applicable six months after the European Commission publishes notice of this confirmation. (6)

\section{CLINICAL REGULATIONS IN USA (7)}

The Food and Drug Administration (FDA or USFDA) is a federal agency of the United States Department of Health and Human Services, one of the United States federal executive departments. The FDA is responsible for protecting and promoting public health through the control and supervision of food safety, tobacco products, dietary supplements, prescription and over-the-counter pharmaceutical drugs (medications), vaccines, biopharmaceuticals, blood transfusions, medical devices, electromagnetic radiation emitting devices (ERED), cosmetics, animal foods \& feed and veterinary products. (5)The FDA was empowered by the United States Congress to enforce the Federal Food, Drug, and Cosmetic Act, which serves as the primary focus for the agency; the FDA also enforces other laws, notably Section 361 of the Public Health Service Act and associated regulations, many of which are not directly related to food or drugs. These include regulating lasers, cellular phones, condoms and control of disease on products ranging from certain household pets to sperm donation for assisted reproduction.

The FDA is led by the Commissioner of Food and Drugs, appointed by the President with the advice and consent of the Senate.

The FDA has its headquarters in unincorporated White Oak, Maryland. The agency also has 223 field offices and 13 laboratories located throughout the 50 states, the United States Virgin Islands, and Puerto Rico. (3) In 2008, the FDA began to post employees to foreign countries, including China, India, Costa Rica, Chile, Belgium, and the United Kingdom.

Clinical trials are conducted by US pharmaceutical and medical device companies in support of an application to the FDA for authorization to market a drug or device. Firstly applicant files the Investigational New Drug applications (INDs) that must be completed to file a New Drug Application (NDA) because most clinical trials conducted by pharmaceutical and biotech companies are undertaken pursuant to an IND.

The federal Food Drug and Cosmetic Act (FD\&C) was passed in 1938 after a tragedy involving a deadly sulfanilamide elixir; it required FDA testing for safety for the first time. In 1962, Congress passed significant amendments to the Act adding a requirement for proof of efficacy before marketing of drugs in response to the thalidomide tragedy.

Section 505 of the FD \& C provides that no person may introduce a new drug into interstate commerce without first filing an application with the FDA. This section of the law specifies the general content of such an application. The FDA promulgated regulations implementing this section of the FD\&C at 21 C.F.R. In 1991, the FDA and the Department of Health and Human Services (DHHS) adopted the so-called "Common Rule" intended to protect the rights of human subjects enrolled in clinical trials. This is codified at 45 C.F.R. Part 46 Subpart A for those clinical studies conducted by or with the support of federal agency or department and at 21 C.F.R.

All clinical trials conducted to secure FDA marketing authorization must adhere to 21 C.F.R. Parts 50 and 56 which are regulations designed to protect the rights of human subjects. Part 50 governs the requirement for securing informed consent from human subjects who participate in clinical trials. This requirement applies not only to drug trials but to any clinical investigation that involves other substances (e.g., food, additives) or medical devices. The general requirement for informed consent is set forth-in 21 C.F.R. Part 50 also includes requirements for documentation of consent and exceptions for the requirement, as well as special safeguards for children and other vulnerable populations.

FDA publishes guidance and information sheets to provide industry and researchers with more information about the agency's views on application of regulations among other topics. The guidance is not binding on the agency but is very helpful to those seeking to conform to the regulations. 
21 C.F.R. Part 56 sets forth the legal obligation to secure review of any clinical investigation subject to FDA regulation by an independent body known as an Institutional Review Board (IRB). Under the requirements set forth in 21 C.F.R. Part 56, the IRB must not only approve the investigation before it can commence but also must monitor it and ensure that it is conducted in accordance with the approval given by the IRB. Part 56 also sets forth requirements for the composition and operation of the IRB. IRBs must meet these requirements to be qualified by the FDA. If the FDA determines that a registered IRB is not adhering to the regulations and that the lack of compliance may adversely affect the rights or safety of human subjects, it may disqualify the IRB. In this case, the IRB may not oversee clinical investigations that support FDA marketing authorization or other action.

21 C.F.R. permits waiver of the requirement for IRB review upon application by the sponsor to the FDA; a waiver may be granted by the FDA in circumstances where the agency determines that the rights of the human subjects can be protected by other mechanisms. These alternative mechanisms include review by an independent ethics committee operated under the regulatory framework of another country that adheres to international standards of clinical practice.

21 C.F.R. Part 54 requires certain disclosures regarding financial relationships between sponsors and investigators as a financial relationship may create a conflict of interest for an investigator that could adversely affect the trial outcome.(7)

\section{CONCLUSION}

Regulatory authorities review and evaluate clinical study data and are responsible for ensuring new medications are safe and effective for their intended use before they are approved for use by patients. If a submission is successful the regulatory authorities will grant marketing authorization (permission to sell the drug).
I take this opportunity to express my deep sense of gratitude to IJDRA Journal for publishing my Article.

\section{CONFLICT OF INTEREST}

The authors declare that there are no conflicts of interest.

\section{REFERENCES}

1. Clinical Trial Phases [Internet]. International Journal of Clinical Medicine; 2014 [Cited 2017 Jan 14]. Available from:

http://www.scirp.org/journal/ijcmhttp://dx.doi.org/10. 4236/ijcm.2014.521175

2. Clinical Trials [Internet]. PubMed Health [Cited 2017 Jan 12]. Available from:

https://www.ncbi.nlm.nih.gov/pubmedhealth/PMH00 63029/

3. F. Hoffmann-La Roche Ltd GPS Public Affairs. Understanding clinical trials [Internet]. Basel, Switzerland. [Cited 2017 Feb 11]. Available from: http://www.roche.com/dam/jcr:1d4d1b52-7e01-43ac862f17bb59912485/en/understanding_clinical trials.pdf

4. Tharani R. An Introduction to Clinical Trial Regulatory Agencies in India [Internet]. [Cited 2017 Feb 12]. Available from:

http://www.pharmainfo.net/book/clinical-pharmacy /introduction-clinical-trial-regulatory-agencies-india

5. European Medicines Agency - Science Medicines Health. [Internet]. [Cited 2017 Feb 04]. Available from:

http://www.ema.europa.eu/ema/index.jsp?curl=pages/ regulation/general/general_content_000629.jsp

6. European Commission - Medicinal Products for Human Use. [Internet]. [Cited 2017 Feb 16]. Available from:

https://ec.europa.eu/health/human-use/clinicaltrials/regulation_en

7. Sherwood, Ted. "Generic Drugs: Overview of ANDA Review Process" [Internet]. CDER Forum for International Drug Regulatory Authorities. [Cited 2017 Feb 08]. Available from:

https://oig.hhs.gov/oei/reports/oei-04-07-00280.pdf

\section{ACKNOWLEDGEMENT}

